\title{
Biofood im Cyberspace
}

\begin{abstract}
Das Internet boomt. Was für Bücher, CDs oder Reisetickets mittlerweile gang und gäbe ist, gilt zunehmend auch für ökologische Produkte. Obwohl die meisten Öko-Internet-Angebote noch den Standards konventioneller Webseiten hinterher hinken, sind beispielsweise Biolebensmittel im Internet keine Ausnahmeerscheinung mehr. Das Internet ermöglicht auch für den Absatz ökologisch erzeugter Lebensmittel erfolgversprechende Szenarien. Die Erfolgsaussichten dieser Ansä̈ze sind umso besser, je klarer biomarktspezifische Lösungen entwickelt und entsprechende Mehrwerte für die jeweilige Zielgruppe angeboten werden.
\end{abstract}

D botschaft ist für die spätere Kaufentscheidung ein wesentlicher Faktor. Insbesondere Anbieter ökologischer Waren sind gefordert, eine Vertrauensbasis zur Zielgruppe aufzubauen und die ökologische Wertigkeit des entsprechenden Produktes glaubhaft zu vermitteln. Grundsätzlich können bei Werbung zwei Dimensionen unterschieden werden: Werbeobjekt und Werbebotschaft. Für das Werbeobjekt lassen sich eine primär unternehmensbezogene und eine primär produktbezogene Werbung differenzieren. Die andere Dimension wird durch die inhaltliche Ausgestaltung der Werbung gebildet - die Werbebotschaften. Deren Pole sind sachliche und emotionale Ausgestaltung (1).

Aufgabe der produktbezogenen Werbung ist es, den Absatz von Produkten zu fördern. Dagegen hat die Unternehmenswerbung zum Ziel, Informationen über das Unternehmen als Ganzes zu vermitteln. Vor allem im Öko-Bereich ist ein Trend zur unternehmensbezogenen Werbung zu verzeichnen. Dies ist insbesondere darauf zurückzuführen, dass dem Ansehen des Unternehmens bei der Kaufentscheidung eine immer größere Rolle zukommt und dieses somit zunehmend einen Erfolgs- und Wettbewerbsfaktor darstellt (2).

Die sachliche Werbung über Information kann als die klassische Form der Positionierung angesehen werden. Ziel ist es, den Konsumenten durch ein umfassendes Informationsangebot eine rationale Beurteilung $\mathrm{zu}$ ermöglichen und produktspezifische Unsicherheiten abzubauen. Sie ist somit für Anbieter ökologischer Produkte gut geeignet. Das Internet bietet hierbei sehr gute Ansatzpunkte, nicht nur mit seiner umfassenden Informationsfunktion (zum Beispiel über E-mail-Newsletter, Links zu Umweltorganisationen), sondern auch durch seine Möglichkeit zur Interaktion und Individualisierung (Communitybildung, direkter Kontakt zum Anbieter).

\section{Mischformen nötig}

Bio-Websites brauchen Mischformen zur Differenzierung. Rein sachliche Werbung verliert angesichts der zunehmenden Sättigung der Märkte und der daraus resultierenden Informationsüberlastung an Bedeutung. Darüber hinaus unterstïtzen Trends wie Hedonismus oder Individualität immer stärker eine Entwicklung hin zum Erlebniskonsum. Unternehmen greifen daher öfter auf emotionale Werbebotschaften zurück, die einen Mehrwert beim Konsumenten in Form eines sogenannten Konsumerlebnisses schaffen (3). Die emotionale Positionierung strebt die Verbindung von Spaß, Genuss und Lebensfreude mit dem umweltbewussten Konsum an. Besonders Kunden ökologisch erzeugter Lebensmittel messen dem Erlebnis Einkauf einen hohen Stellenwert bei. So ist Naturkostkunden der Genuss- und Spaßaspekt beim Einkauf wichtig (4). Auch im Internet ist das Einkaufserlebnis ein entscheidender Faktor (5). Nicht nur die Höhe der Ausgaben für den Einkauf lässt sich positiv beeinflussen, sondern auch die Kundenbindung. Der Erlebnisaspekt trägt folglich mit zur Umsatzstärkung bei.

Aus ökologischer Perspektive sind vor allem Mischformen für das Internet interessant. Es gilt das Prinzip: „Appeliere an ein Bedürfnis und informiere über Eigenschaften eines

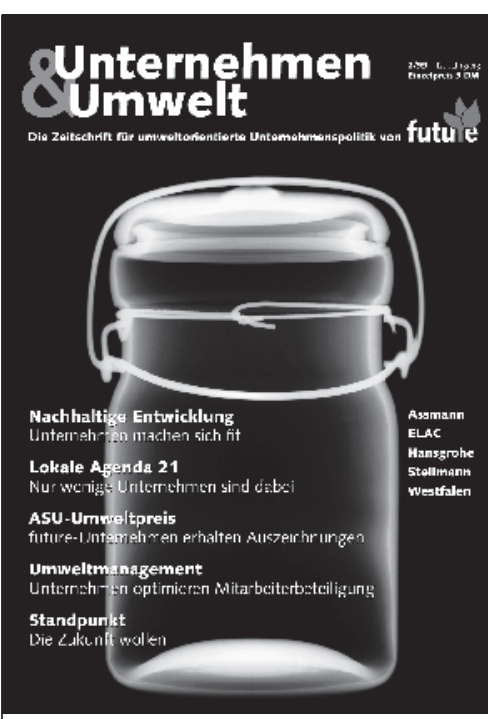

Unternehmen \& Umwelt

- ist die Zeitschrift für umweltorientierte Unternehmenspolitik von future e.V.,

- berichtet über den neuesten Stand zum betrieblichen Umweltmanagement in Theorie und Praxis,

- bietet vier Mal im Jahr ein Schwerpunktthema, Unternehmensbeispiele, Literaturund Veranstaltungshinweise und aktuelle future-Nachrichten.

Zum Beispiel Heft 2/99:

Nachhaltigkeit

- Nachhaltige Entwicklung: Unternehmen machen sich fit.

- Lokale Agenda 21: Nur wenige Unternehmen sind dabei.

- Betrieblicher Umweltschutz: Assman, ELAC, Hansgrohe, Steilmann.

- Umweltmanagement: Unternehmen optimieren die Mitarbeiterbeteiligung.

Fordern Sie Ihr kostenloses Probeexemplar an: Fon: 0234 - 97995 - 13 Fax: 0234 - 97995 - 14

future e.V. Büro Bochum Am Varenholt 123 44797 Bochum email: future@umis.de 


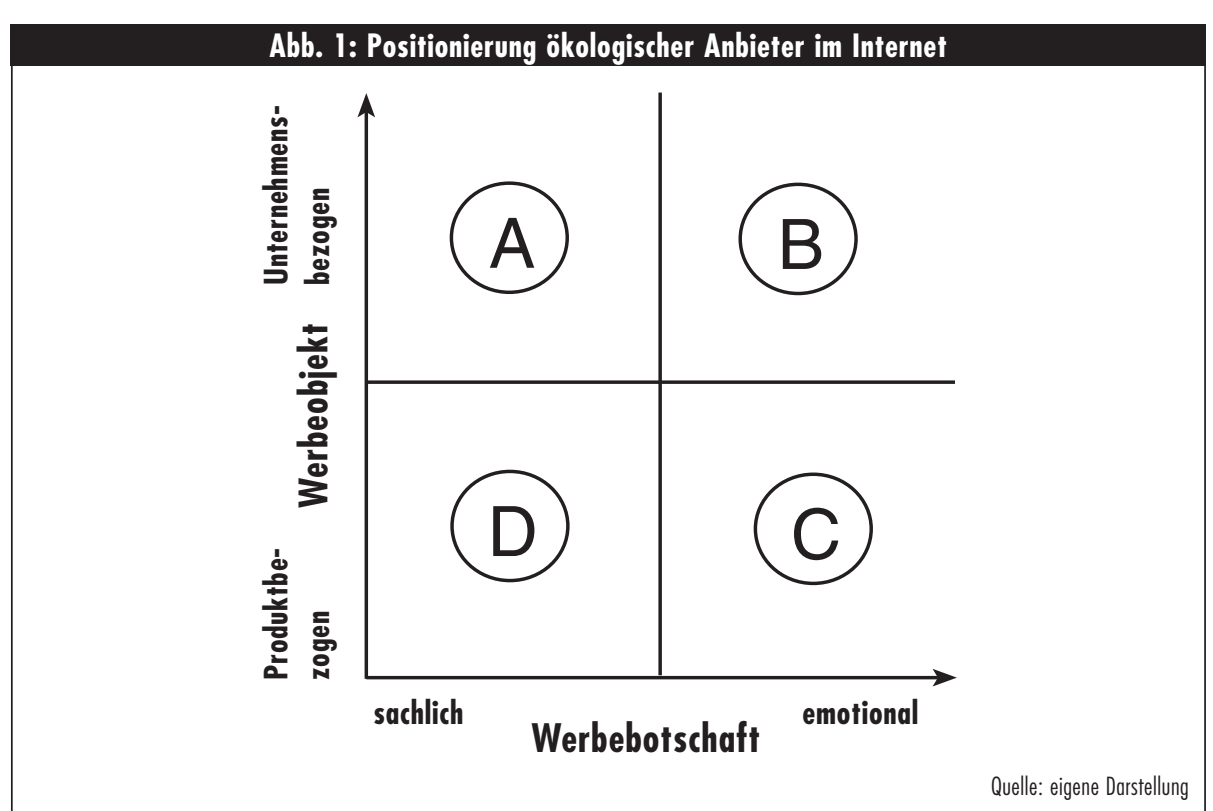

Gegenstandes, die dazu dienen, dieses Bedürfnis zu befriedigen." (6) Ziel der informativen Elemente ist es, Vertrauen zu gewinnen und ein positives ökologisches Image aufzubauen, um damit Wiederkäufer zu gewinnen. Hingegen zielt die emotionale Komponente darauf $a b$, Umsatz durch erhöhte Kundenbindung bzw. Impulskäufe oder Wiederkehr der Kunden zu erlangen. Beides führt zu neuen Marktpotenzialen für Bioanbieter.

\section{- Vier Positionierungsansätze}

Welche Positionierungsansätze nutzen ökologische Anbieter im Internet? Zur Beantwortung dieser Frage wurden in einer Untersuchung des Internet-Status im Umfeld von Produktion und Handel mit ökologisch erzeugten Lebensmitteln 230 Internetseiten begutachtet (7). Folgende Ansätze ließen sich identifizieren (vgl. Abb. 1):

- Typus A: Sachlich und unternehmensbezogene Positionierung (Beispiel: http://www.

hipp.de): Der Internetauftritt des Unternehmens zeichnet sich vor allem durch die Sachlichkeit und den Umfang der vermittelten Informationen aus. Es reicht von einer ausführlichen Darstellung zu Bio-Qualität bei Hipp über einen Ratgeber für Mütter bis hin zu Unternehmensinformationen wie Umweltmanagement und Öko-Bilanz. Dieser Wissensfundus wird durch interaktive Elemente wie Newsletter und individuelle OnlineBeratung ergänzt.

- Typus B: Emotional und unternehmensbezogene Positionierung (Beispiel: http:// www.coop.ch/naturaplan): Die Vermittlung von
Image und Lebensgefühl steht für die Bio-Produktreihe Coop-NATURAplan im Mittelpunkt. Dazu werden weniger klare Positionen kommuniziert, als vielmehr an Emotionen appelliert. Als Medien werden dazu unterschiedlichste Ansätze genutzt: ein Online-Spiel, ein Fitnesstest, eine Coop-NATURAplan-Entdekkungsreise oder vom Internetnutzer steuerbare Live-Kameras mit Bildern von ausgewählten Biobetrieben.

Typus C: Emotional und produktbezogene Positionierung (Beispiel: http://www.viana. de): Als Aufhänger der Internetpräsentation der Viana Naturkost GmbH wurde das Produkt Tofustäbchen in den Mittelpunkt gerïckt. Mit Animationen wie einem über das Meer fahrenden Schiff und der Verwendung von Kapitän ,Tofu' werden nur indirekt Produkteigenschaften angesprochen - vielmehr wird auf die Wünsche des vor allem jüngeren Publikums eingegangen und so ein eigenständiges Produkt-Image entwickelt.

Typus D: Sachlich und produktbezogene Positionierung (Beispiel: http://www.zwoelbe rich.de): Das Angebot an ökologischen Spirituosen wird klar strukturiert im Internet präsentiert. Bereits auf der Einstiegsseite ist eine Auswahl an unterschiedlichen Weiß-, Rot- und Schaumweinen sowie Likören und Destillaten dargeboten. $\mathrm{Zu}$ jeder Produktgruppe lassen sich per Mausklick detaillierte Informationen zu Geschmack, Herstellung und Prämierungen abrufen. Unternehmensinformationen rücken zugunsten der detaillierten und graphisch ansprechenden Präsentation der Weine in den Hintergrund.
Die beschriebenen Positionierungsansätze definieren den Entwicklungsrahmen für biospezifische Internetangebote. Die erwähnten Beispiele zeigen dabei erste Ansätze für einen kreativen Umgang mit dem Medium Internet im Biobereich. Im Hinblick auf mögliche Potenziale für eine biospezifische Internet-Entwicklung ist vor allem interessant, dass die Möglichkeiten, sich von konventionellen Angeboten abzugrenzen, bislang kaum genutzt werden. Große Chancen liegen für Bio-Anbieter vor allem in der Kombination von sachlichen Argumenten und der Nutzung emotionaler Mehrwerte: Während ein umfassendes und individualisiertes Informationsangebot Vertrauen beim Konsumenten schafft, führen emotionale Mehrwerte zur Kundenbindung und Wiederkäufen.

\section{Anmerkungen}

(1) Vgl. Kroeber-Riel, W.: Konsumentenverhalten, München 1999.

(2) Vgl. Hopfenbeck, W./ Roth P.: Öko-Kommunikation: Wege zu einer neuen Kommunikationskultur, Landsberg/ Lech 1994.

(3) Weinberg, P.: Erlebnismarketing, München 1992. (4) Vgl. Kutz, K.: On-line Grocery Shopping on Track for Rapid Growth, Andersen Consulting Newsletter January 20th, Chicago 1998.

(5) Vgl. BNN: Den Kunden im Blick - Kundenumfrage zu ökologischen Lebensmitteln und deren Verkaufsstellen, Köln 1999.

(6) Kroeber-Riel, W.: Strategie und Technik der Werbung, Stuttgart 1990.

(7) Vgl. zu den detaillierten Ergebnissen Nachtmann, M./ Kolibius, M.: Biofood im Cyberspace - Electronic Commerce am Beispiel ökologisch erzeugter Lebensmittel, Berlin 2000 (in Vorbereitung).

\section{Die Autoren}

Mischa Kolibius ist wissenschaftlicher Mitarbeiter am Institut für Wirtschaft und Ökologie an der Universität St. Gallen (IWÖ-HSG), Matthias Nachtmann ist Doktorand an der Universität Oldenburg und Berater bei der Management- \& Technologieberatung Gora, Hecken \& Partner.

Kontakt: IWÖ-HSG, Tigerbergstr. 2, CH-9000 St. Gallen, Tel. 0041-71-224-2589, Fax -2722, E-mail: Mischa.Kolibius@unisg.ch; Matthias Nachtmann, Wintergasse 13, 65239 Hochheim, E-mail: mnachtmann@aol.com 
(c) 20I0 Authors; licensee IÖW and oekom verlag. This is an article distributed under the terms of the Creative Commons Attribution Non-Commercial No Derivates License (http://creativecommons.org/licenses/by-nc-nd/3.o/), which permits unrestricted use, distribution, and reproduction in any medium, provided the original work is properly cited. 